\title{
Índice Cefálico en un Grupo de Individuos Mapuches de la IX Región de Chile
}

\author{
Cephalic Index in a Group of Mapuche Individuals in the IX Region of Chile \\ Mariano del Sol
}

DEL SOL, M. Indice cefálico en un grupo de individuos mapuches de la IX Región de Chile. Int. J. Morphol., 23(3):241-246, 2005.

RESUMEN: El grupo étnico mapuche tiene una amplia distribución de asentamiento en la IX Región de Chile, estando más aislados de centros urbanos aquellos individuos que viven en algunos sectores del litoral de la Región. Varios factores, entre éstos los aspectos étnicos son considerados determinantes en la forma de la cabeza y en sus índices.

Se efectuó un estudio antropométrico en 50 individuos adultos (19-83 años), de sexo masculino, del grupo étnico mapuche, pertenecientes a algunas reducciones de la zona costera de la IX Región de Chile. En ellos se midieron los diámetros cefálicos y curvas craneales y se determinó el índice cefálico.

El diámetro occipitofrontal máximo promedio fue de $184 \mathrm{~mm}$ (DS 6), con límites superior de $185 \mathrm{~mm}$ e inferior de $182 \mathrm{~mm}$. El diámetro transverso máximo promedio fue de $148 \mathrm{~mm}$ (DS 4), con límites superior de 149mm e inferior 140mm. Las curvas craneales fueron, en promedio: anteroposterior $395 \mathrm{~mm}$ (DS 13), supraauricular $315 \mathrm{~mm}$ (DS 12) y circunferencia máxima 632 mm (DS 20). Los individuos eran mesocéfalos en un $66 \%$, braquicéfalos el 28\%, hiperbraquicéfalos el $4 \%$ y dolicocéfalos el 2\%. El índice cefálico promedio fue de 80,4 .

Los individuos del grupo étnico mapuche, presentan características típicas de mesocefalia, con una clara tendencia a la braquicefalización. Los datos anatómicos y antropológicos obtenidos, aportarán nuevos conocimientos a la Antropología Biológica y Física y a los profesionales del área de la Salud.

PALABRAS CLAVE: Índice cefálico; Grupo étnico Mapuche; Chile.

\section{INTRODUCCIÓN}

El cráneo del Hombre ha sido estudiado en todos los tiempos desde el punto de vista descriptivo, siendo más recientes los estudios comparativos, que han permitido determinar características propias, en relación con la raza, grupo étnico, sexo, edad, entre otras.

Para conseguir una evaluación racial más objetiva, durante mucho tiempo, fueron practicados estudios métricos. Técnicas internacionalmente aceptadas de craniometría otorgaron una gran cantidad de datos étnicos comparables para los hombres y, en una extensión menor, para las mujeres.

El crecimiento en el ancho de la cabeza ocurre en las suturas sagital, esfenofrontal, esfenotemporal y occipitomastoidea y en las articulaciones petro-occipitales, a diferencia del crecimiento en altura que ocurre en las suturas frontocigomáticas y escamosa, en el pterio y el asterio.
De acuerdo a lo planteado por Williams et al., 1995, la forma de la cabeza, o de la bóveda cráneana, no está relacionada con el crecimiento del cerebro, pero sí con factores genéticos, lo que está apoyado por la gran gama de índices y formas craneales en los grupos raciales.

Ferembach (1956), en un extenso estudio (Memoria original) sobre las constantes craneanas, braquicráneo y arquitectura craneana, señaló que la braquiocefalia apareció en el Mesolítico en una parte de Europa y también en Africa del Norte. Sin embargo, la teoría de la migración de población amarilla no puede explicar la expansión de la braquiocefalia en Europa. Esto se produciría por la influencia de los músculos, la presión sobre el cráneo durante la infancia, la talla y la sobre o deficiente alimentación. 
Según Verdun et al. (1958) la hiperdolicocefalia es habitualmente el signo de cruzamiento de dos razas de dolicocéfalos, mientras que la hiperbraquicefalia indica cruzamiento de dos razas braquicefálicas. La mesocefalia en cambio, es signo habitual de cruzamiento de dos razas de índices cefálicos opuestos.

Considerando por una parte, que el grupo étnico mapuche tiene una amplia distribución de asentamiento, bajo el sistema reduccional, en la IX Región de Chile y, por, otra el gran desarrollo que ha tenido la Antropología Física y Biológica, llama la atención la poca información que se puede encontrar sobre este grupo. Sin embargo, se pueden encontrar en la literatura algunos estudios que tratan aspectos anátomo-antropológicos destacándose las investigaciones de Latcham (1911); Guevara (1927); Henckel (1958); del Sol \& Henríquez (1985); del Sol et al. (1990), del Sol \& Olave (1996 y 1998), Soto (2002) y recientemente un estudio postural efectuado por del Sol \& Hunter (2004).

Algunos investigadores como Gavrilovic (1967), han señalado que el índice cefálico ha sido utilizado de manera indiscriminada como medio para clasificar a los diferentes grupos étnicos, y que no sería válido porque estos grupos no existirían totalmente puros. A pesar de ello, siempre resulta importante conocer parámetros anatómicos y antropométricos, no sólo para contribuir con la Antropología Anatómica, Biológica o Física, sino también para aportar nuevos datos a los profesionales de la salud, en especial, de los individuos pertenecientes al mayor grupo étnico de Chile.

\section{SUJETOS Y MÉTODO}

Es conocido que el índice cefálico se fija en el momento de la pubertad, siendo como los demás caracteres craneales, menos fijo en la mujer. Por lo anterior, se prefirió efectuar el estudio de los índices cefálicos solamente en individuos de sexo masculino, por ser éstos más homogéneos.

El estudio se realizó en 50 individuos adultos (entre 19 y 83 años), de sexo masculino, del grupo étnico mapuche, provenientes de las reducciones ubicadas en la zona costera de la IX Región de Chile, en el paralelo 38, entre los $20^{\prime}$ y $50^{\prime}$.

Los individuos aceptaron voluntariamente participar de la entrevista. Para cada uno de ellos, se elaboró una ficha registrándose en ella las siguientes mediciones craneales:
1. Diámetros anteroposteriores: Occipitomentoniano, occipitofrontal, suboccipitofrontal y suboccipitomentoniano. El diámetro utilizado para determinar el índice cefálico fue el anteroposterior máximo o longitud máxima del cráneo, es decir, el mayor diámetro en el plano mediano, determinado por delante por la glabela y por detrás por el punto más saliente por arriba del foramen magno.

2. Diámetros transversales: Biparietal y bitemporal. Al igual que en el caso anterior, el diámetro transversal máximo de la cabeza es el mayor diámetro horizontal y transversal que se pudo medir y coincidió con el diámetro biparietal.

3. Curvas craneales: Sagital o anteroposterior mediana, supraauricular y horizontal o circunferencia máxima. Esta última curva pasa, por delante, por encima de los arcos ciliares y por detrás por el occipital. La curva sagital se tomó entre el nasion por delante y un punto entre el inion y el opistion por atrás. La curva supraauricular se tomó en el plano verticotransversal en las partes más sobresalientes de la raíz cigomática posterior, exactamente por arriba del meato acústico externo. Las curvas craneales fueron tomadas usando una huincha métrica de alta precisión.

El índice cefálico (en el ser humano vivo), al igual que el índice craneano (en el hueso seco) presenta grandes variaciones. Todas las variaciones son divididas arbitrariamente en escalas, cubriendo secciones del $5 \%$ del total de la variación. Este índice fue calculado dividiendo el diámetro transverso máximo por el diámetro anteroposterior máximo, multiplicado por 100.

Se utilizó la siguiente clasificación: Dolicocéfalos, mesocéfalos y braquicéfalos. Para valores extremos: hiperdolicocéfalos e hiperbraquicéfalos.

\section{RESULTADOS}

Los resultados de la mediciones de los diámetros cefálicos, anteroposteriores y transversales, determinados en 50 individuos del grupo étnico mapuche se muestran en las Tablas I y II. Las mediciones de las curvas craneales se presentan en la Tabla III.

El índice cefálico promedio fue de 80,4 (DS. 2), con características de mesocefalia y tendencia a la braquicefalización. El índice cefálico mínimo calculado en un individuo fue de 75,0 y el máximo observado en la muestra fue de 88,2. El número de individuos y el porcentaje relativo a las diversas clasificaciones cefálicas de ellos, son presentados en la Tabla IV. 
Tabla I. Diámetros cefálicos anteroposteriores de 50 individuos mapuches, adultos, de sexo masculino, de la IX Región de Chile.

\begin{tabular}{lcccc}
\hline & Occipitomentoniano & Occipitofrontal & Suboocipitofrontal & Suboccipitomentoniano \\
Promedio & 205 & 184 & 178 & 157 \\
Desviación Estándar & 13 & 6 & 7 & 7 \\
Error Estándar & 2 & 1 & 1 & 1 \\
L. Inf. (95\% conf.) & 202 & 182 & 176 & 155 \\
L. Sup. (95\% conf.) & 209 & 185 & 179 & 159 \\
Mínimo & 180 & 170 & 150 & 140 \\
Máximo & 236 & 200 & 198 & 175 \\
p25 & 200 & 180 & 175 & 154 \\
p50 & 206 & 184 & 178 & 158 \\
p75 & 210 & 187 & 180 & 160 \\
\hline
\end{tabular}

Tabla II. Diámetros cefálicos laterolaterales (transversales) de 50 individuos mapuches, adultos, de sexo masculino, de la IX Región de Chile.

\begin{tabular}{lcc}
\hline & Biparietal (mm) & Bitemporal (mm) \\
Promedio & 148 & 141 \\
Desviación Estándar & 4 & 4 \\
Error Estándar & 1 & 1 \\
L. Inf. (95\% conf.) & 146 & 140 \\
L. Sup. (95\% conf.) & 149 & 142 \\
Mínimo & 140 & 130 \\
Máximo & 156 & 150 \\
p25 & 145 & 138 \\
p50 & 149 & 141 \\
p75 & 150 & 144 \\
\hline
\end{tabular}

Tabla IV. Indice cefálico de 50 individuos mapuches, adultos, de sexo masculino, de la IX Región de Chile.

\begin{tabular}{lrr}
\hline Hiperdolicocéfalos (bajo 70,9) & \multicolumn{1}{c}{-} \\
Dolicocéfalos $(71,0-75,9)$ & 1 & $2 \%$ \\
Mesocéfalos $(76,0-80,9)$ & 33 & $66 \%$ \\
Braquicéfalos $(81,0$ a 85,4$)$ & 14 & $28 \%$ \\
Hiperbraquicéfalos (superior a 85,5) & 2 & $4 \%$
\end{tabular}

Tabla III. Curvas craneales de 50 individuos mapuches, adultos, de sexo masculino, de la IX Región de Chile.

\begin{tabular}{lccc}
\hline & $\begin{array}{c}\text { Anteroposterior } \\
(\mathrm{mm})\end{array}$ & $\begin{array}{c}\text { Supraauricular } \\
(\mathrm{mm})\end{array}$ & $\begin{array}{c}\text { Circunferencia } \\
\text { Máxima }(\mathrm{mm})\end{array}$ \\
Promedio & 395 & 315 & 632 \\
Des viación Estándar & 13 & 12 & 20 \\
Error Estándar & 2 & 2 & 3 \\
L. Inf. (95\% conf.) & 391 & 312 & 626 \\
L. Sup. (95\% conf.) & 398 & 318 & 637 \\
Mínimo & 363 & 285 & 543 \\
Máximo & 422 & 350 & 675 \\
p25 & 389 & 307 & 625 \\
p50 & 395 & 318 & 634 \\
p75 & 400 & 320 & 640 \\
\hline
\end{tabular}




\section{DISCUSIÓN}

Es conocido que uno de los factores que influyen en las variaciones anatómicas son, entre otros, los grupos étnicos. La evolución del Hombre ha permitido un aumento progresivo de la braquicefalia. Por otra parte, es muy difícil encontrar etnias puras y, por tanto su valor, clasificatorio cada día será de menor importancia.

En la Tabla V se puede observar la comparación entre algunos estudios realizados, en diferentes grupos étnicos, de los diámetros cefálicos (anteroposterior y transversal), y la determinación de los valores del índice cefálico.

García \& Lips (1986a) determinaron el índice cefálico en un grupo de individuos chilenos, adultos y de distintas regiones del país. Encontraron que el 39,8\% de ellos eran mesocéfalos, el 35,2\% braquicéfalos, el $17 \%$ hiperbraquicéfalos y sólo el $7 \%$ dolicocéfalos. Estos mismos autores (1986b) estudiaron las variaciones del índice cefálico en chilenos según ascendencia, observando valores medios del índicce cefálico en chilenos de 81,51 $\pm 0,46$, en hombres sin ascendencia europea de 82,23 $\pm 0,63$, con ascendencia europea mediterránea $81,19 \pm 0,95$ y con ascendencia europea nórdica coorespondía a 79,72 $\pm 0,79$. Comprobaron mayores braquicefalia e hiperbraquicefalia en los individuos sin ascendencia europea, siendo predominante la mesocefalia en los individuos con ascendencia europea mediterránea.

De acuerdo a lo observado por Olivier \& Kauffmann, (1955), los Naga, uno de los siete grupos que viven en Assam, la provincia más oriental de la India, un enclave entre el Tibet al Norte, la China al Este, Birmania al Sur y Bengala al Oeste, son fracamente mesocéfalos, existiendo sin embargo, diferencias significativas incluso entre tribus.
Estudios realizados por Lehmann \& Marquer (1960) en indios del grupo «Guambiano-kokonuko», que viven en el Suroeste de Colombia y que comprenden varios grupos correspondientes a antiguas tribus, determinaron que la cabeza de estos individuos es relativamente poco desarrollada en el sentido longitudinal, pero con una extensión más marcada en el diámetro transverso. Así, estos individuos presentan una fuerte mesocefalia al límite de la braquicefalia, similar a los observado en mapuches. Los extremos en ambos grupos implican una variedad normal que va desde la dolicocefalia a la hiperbraquicefalia.

El índice cefálico de un grupo de hombres de la isla de la Tortuga en Haití, según Benoist (1962), era ampliamente mesocefálico. Estudios recientes de Shah \& Jadhav (2004), realizados en 302 estudiantes de Medicina de 17 a 23 años, en la India, determinaron un índice cefálico promedio de 80.42, es decir, con características de mesocefalia.

Los Fang, el grupo étnico más importante de la población de Africa Central, que constituyen un tercio de Gabón y algunas partes meridionales de Camerún, presentan un índice cefálico de 77,5, clasificándose como mesocefálicos. Los individuos de gran altura son más dolicocéfalos (Lalouel, 1957). Similar a lo observado en la población Corsa por Piquet-Thepot (1962) quien determinó que los individuos estudiados presentanan una fuerte mesocefalia asociada a la gran altura de la cabeza.

La braquialización de la cabeza ha ido en aumento en el mundo, señalándose que ésta es mayor en la razas amarilla y menor en la blanca y negra, siendo esta última la que presenta mayor dolicocefalia. A pesar de lo anterior, dentro de

Tabla V. Comparación de los diámetros cefálicos, anteroposterior máximo y transverso máximo, en estudios realizados en diferentes razas y grupos étnicos.

\begin{tabular}{|c|c|c|c|c|c|c|c|c|}
\hline $\begin{array}{l}\text { Grupo étnico } \\
\text { Investigadores }\end{array}$ & $\begin{array}{l}\text { Mapuche } \\
\text { Del Sol }\end{array}$ & $\begin{array}{l}\text { Naga (India) } \\
\text { Olivier \& } \\
\text { Kauffmann }\end{array}$ & $\begin{array}{l}\text { Chileno } \\
\text { García \& } \\
\text { Lips (1) }\end{array}$ & $\begin{array}{l}\text { Guambiano } \\
\text { Kokonuko } \\
\text { Lehmann \& } \\
\text { Marquer }\end{array}$ & $\begin{array}{l}\text { Fang } \\
\text { (Africa } \\
\text { Central) } \\
\text { Lalouel }\end{array}$ & $\begin{array}{l}\text { De Isla } \\
\text { Tortuga } \\
\text { (Haití) } \\
\text { Benoist }\end{array}$ & $\begin{array}{l}\text { Esquimal } \\
\text { Angmassalik } \\
\text { Gessain }\end{array}$ & $\begin{array}{l}\begin{array}{l}\text { Aymara y } \\
\text { boliviano }\end{array} \\
\text { Ruffié et al. }\end{array}$ \\
\hline $\begin{array}{l}\text { Diámetro } \\
\text { anteroposterior } \\
\text { máximo }\end{array}$ & $\begin{array}{l}184 \pm 6 \\
170-200 \\
\text { EE } 2\end{array}$ & $\begin{array}{l}187,5 \pm 5,71 \\
174-200\end{array}$ & $189,8 \pm 0,7$ & $\begin{array}{l}188 \\
170-207\end{array}$ & $\begin{array}{l}193 \\
184-200\end{array}$ & $\begin{array}{l}187,24 \quad \pm \\
0,98 \\
172-198 \\
\text { EE 6,34 }\end{array}$ & $\begin{array}{l}193,7 \pm 0,30 \\
181-206\end{array}$ & $\begin{array}{l}180,7 \\
184,98 \\
\text { EE } 17.2 \\
\text { EE } 10,24\end{array}$ \\
\hline $\begin{array}{l}\text { Diámetro } \\
\text { transversal } \\
\text { máximo }\end{array}$ & $\begin{array}{l}148 \pm 4 \\
140-156 \\
\text { EE } 1\end{array}$ & $\begin{array}{l}146,9 \pm 4,59 \\
133-159\end{array}$ & $154,5 \pm 0,7$ & $\begin{array}{l}155 \\
134-163\end{array}$ & $\begin{array}{l}14,96 \\
140-155\end{array}$ & $\begin{array}{l}147,02 \quad \pm \\
0,77 \\
140-158 \\
\text { EE } 5,02\end{array}$ & $\begin{array}{l}147,4 \pm 0,24 \\
136-160\end{array}$ & $\begin{array}{l}154,6 \\
151,52 \\
\text { EE 8,22 } \\
\text { EE 5,89 }\end{array}$ \\
\hline $\begin{array}{l}\text { Indice cefálico } \\
\text { promedio }\end{array}$ & $\begin{array}{l}80,4 \pm 2 \\
75,0-88,2 \\
\text { EE } 0\end{array}$ & $\begin{array}{l}78,25 \pm 3,06 \\
69,5-86,9\end{array}$ & $81,5 \pm 0,46$ & $\begin{array}{l}80,1 \\
71,4-88,7\end{array}$ & 77,5 & $\begin{array}{l}78,6 \pm 0,56 \\
74,22-88,2 \\
\text { EE } 3,68\end{array}$ & $\begin{array}{l}76,1 \pm 0,33 \\
70-82,5\end{array}$ & \\
\hline
\end{tabular}


cada grupo y, debido a los factores de variación, incluso individual, suelen existir individuos que presentan los diversos tipos de clasificaciones.

Para una estatura media elevada, alrededor de $176 \mathrm{~cm}$, el índice de los Fang baja a 75,8. Los sujetos más altos son francamente dolicocéfalos. Por otra parte, los sujetos más bajos son mesocéfalos, presentando una ligera tendencia a la braquicefalia, hecho que se ha manifestado en casi la totalidad de los grupos étnicos.

Como se puede notar, grupos de individuos tan distintos y de diversas latitudes como los Naga, GuambianoKokonuko, Mapuche y otros, presentan una franca mesocefalia con tendencia a la braquicefalia.

Por el contrario, Gessain (1958), quien estudió las características antropológicas de un grupo de esquimales de Angmassalik, o Ammassalimiut, los cuales viven en tres fiordos en la costa Este de Groenlandia, determinó que éstos eran dolico-mesocéfalos. Ellos tenían el índice cefálico más pequeño y por esta dolicocefalia recibieron el nombre de "especialización" de los esquimales del Este. Valores muy similares de índice cefálico fueron encontrados por Bhatia et al. (1955) en un grupo de 806 individuos del Norte de la India, siendo 155 hiperdolicocéfalos, 19,2\% (64-70), 472 dolicocéfalos, 58,5\% (71-75), 171 sujetos mesocéfalos (76$80)$ y solamente 6 sujetos braquicéfalos (81-86).

Otro de los aspectos a considerar en el índice cefálico, es la evolución de los caracteres morfológicos en función de la edad. Así, Marquer \& Chamla (1961) determinaron, en más de dos mil franceses de 20 a 91 años de edad, que la repartición de dolicocéfalos, mesocéfalos y de braquicéfalos se presentaba relativamente homogénea con la edad. Existía una dominancia en los porcentajes de los mesocéfalos y braquicéfalos, tendencia a equilibrarse entre 40 y $50 \%$, en detrimento de los dolicocéfalos, en alrededor de un $10 \%$.

De acuerdo a la hipótesis que argumentan que el cráneo se tornaría dolicocéfalo con la edad, es posible que así sea, pero muy tardíamenente. En el grupo Mapuche estudiado no fue posible determinar este fenómeno. Pero sí fue posible constatar la Ley de Pitard sobre la braquialización de los individuos de menor talla al interior de los grupos.

Ruffié et al. (1966), en un estudio en 131 sujetos de sexo masculino (19-26 años de edad), 49 Aymara considerados puros y 82 bolivianos, mestizos según los investigadores pero, que ellos se pretendían puros, concluyeron que no existían diferencias significativas entre ambos grupos.

Las opiniones sobre la importancia de determinar el índice cefálico son bastante contradictorias. Como lo indicó Gavrilovic, no sería válido porque no existirían etnias totalmente puras. Por el contrario, Shah \& Jadhav señalaron que el índice cefálico es un importante parámetro para decidir la raza y sexo de un individuo, del cual su identidad no es conocida. Son dos extremos de opinión para un mismo parámetro antropológico. Sin embargo, siempre resultará importante conocer parámetros anatómicos y antropométricos, no sólo para contribuir con la Antropología Biológica o Física, sino también para aportar nuevos datos a los profesionales de la salud y medicina forense del mayor grupo étnico de Chile.

DEL SOL, M. Cephalic index in a group of native Mapuche individuals in the IX Region of Chile. Int. J. Morphol., 23(3):241-246, 2005.

SUMMARY: Native Mapuches live mostly in the Chilean Araucania in the IX Region of the country, being most isolated those living along the coastline. Several factors are considered to be determining in their head shape and indexes, among which ethnic aspects can be pointed out.

Fifty male native Mapuche individuals, all adults living in the coastal range of the IX Region of Chile, with ages ranging between 19-83 years, were anthropomorphically studied. Their cephalic diameters were measured and their cranial index determined.

The mean of the greatest anteroposterior diameter (frontal-occipital) was 184mm (DS6) with superior limits of $185 \mathrm{~mm}$ and inferior $182 \mathrm{~mm}$. The mean of the transverse maximum diameter was $148 \mathrm{~mm}$ (DS4) with superior limits of $149 \mathrm{~mm}$ and inferior 140 $\mathrm{mm}$. The mean of the cranial curves were: anterior-posterior $395 \mathrm{~mm}$ (DS13), supra auricular $315 \mathrm{~mm}$ (DS12) and maximum circumference $632 \mathrm{~mm}$ (DS20). The $66 \%$ of the individuals were mesocephalic, $28 \%$ brachycephalic, $4 \%$ hyperbrachycephalic and $2 \%$ dolichocephalic. The mean of cephalic index was 80.4.

Native Mapuche individuals present typical mesocephalic characteristics and a clear tendency to brachycephalization. The data obtained, anatomical and anthropological, will add up to Biological and Physical Anthropology and benefit the knowledge of health professionals.

KEY WORDS: Cephalic index; Mapuche ethnic group; Chile. 


\section{REFERENCIAS BIBLIOGRÁFICAS}

Bhatia, M.; Thin, J.; Debray, H. \& Cabanes, J. Étude anthropologique et genétique de la population du Nord de l'Inde. Bull. et Mém. Soc. d'Anthrop. de Paris, 10(6):199213, 1955.

Benoist, J. Anthropologie physique de la population de l'ile de la tortue (Haïti) Contribution a l'étude de l'origine des noirs des Antilles Françaises. Bull. et Mém. Soc. d'Anthrop. de Paris, 11(3):315-35, 1962.

Del Sol, M. \& Henríquez, P. J. Evaluación de algunos índices antropométricos de un grupo de población Mapuche. Bol. Mus. Reg. Araucanía, 2:79-89, 1985.

Del Sol, M.; Vieira, M C. \& Olave, E. Estudo morfométrico do esterno no grupo étnico Mapuche. Arq. Anat. Antropol., 41:211-6, 1990.

Del Sol, M. \& Olave, E. Índices claviculares en el grupo étnico Mapuche. Rev. Chil. Anat., 14(2):183-88, 1996.

Del Sol, M. \& Olave, E. El proceso xifoides del esternón mapuche. Rev. Chil. Anat., 16(1):101-6, 1998.

Del Sol, M. \& Hunter, K. Evaluación postural de individuos mapuche de la zona costera de la IX Región de Chile. Int. J. Morphol., 22(4):339-342, 2004.

Ferembach, D. Mémoires Originaux. Constantes craniennes, brachycranie et architecture cranienne. Bull. et Mém. Soc. d'Anthrop. de Paris, 10(7):1-129, 1956.

García, H. F. \& Lips, M. W. Contribución al estudio del índice cefálico en chilenos. An. Anat. Normal., 4:120-3, 1986 .

García, H. F. \& Lips, M. W. Variaciones del índice cefálico en chilenos según ascendencia. An. Anat. Normal., 4:117-9, 1986b.

Gessain, R. Les Eskimo d'Angmassalik principaux caractères anthropologiques. L'Antropologie, 62(5-6):452-84, 1958.

Gravilovic, Z. L'indice cephalique chez le yougoslaves. Comp. Rend. Assoc. Anat., 138:552-8, 1967.

Guevara, T. La complexión física del araucano. In: Historia de Chile; Chile Prehispánico, Balcells, Santiago de Chile, 1927. V 2. pp321-9.

Henckel, C. Antropología Física de los mapuches. Rev. Universitaria, 43(22):13-22, 1958.

Lalouel, J. Anthropométrie des Fang. Bull. et Mém. Soc. d'Anthrop. de Paris, 10(8):371-82, 1957.
Latcham, R. Antropología chilena. I Congreso Científico Panamericano. Santiago, Chile. Ciencias Naturales, Antropología y Etnografía, 3:24-84, 1911.

Lehmann, H. \& Marquer, P. Étude anthropologique des indiens du groupe Guambiano-Kokonuko (Région de Popayán, Colombie). Bull. et Mém. Soc. d'Anthrop. de Paris, 11(1):177240, 1960.

Marquer, P. \& Chamla, M. C. L'evolution des caractères morphologiques en fonction de l'age, chez 2089 français, de 20 a 91 ans. Bull. et Mém. Soc. d'Anthrop. de Paris, 11(2):178, 1961.

Olivier, G. \& Kauffmann, H. E. Anthropologie des Naga chasseurs de têtes de L'Assam. Bull. et Mém. Soc. d'Anthrop. de Paris, 10(6):373-417, 1955.

Piquet-Thepot, M. Étude antrropologique de la population Corse. Bull. Memoires Sociéte d'antropologie de Paris, 11(3):22934, 1962.

Ruffié, Th.; Fernet, M. P. \& Larrouy, G. Étude biométrique du massif facial et de la denture des amérindiens Aymara et des Métis de l'altiplano bolivien. Bull. et Mém. Soc. d'Anthrop. de Paris, 11(9:109-16, 1966.

Shah, G. V. \& Jadhav, H. R. The study of cephalic index in students of Gujarat. J. Anat. Soc. India., 53(1):25-6, 2004.

Soto, M. P. Stature et endogamie dans la population mapuche du Chili. Biométrie Humaine et Anthropologie, 20(3-4):235-46, 2002.

Verdun. J.; Taille, J. Bourdiol, R. \& Poggi, J. Contribution a l'étude de l'anthropologie raciale de la population présente de la France. Bull et Mém. Société Anthrop. de Paris, 10(9):227-44, 1958.

Williams, P. L.; Warwick, R.; Dyson, M. \& Bannister, L. H. Gray Anatomia. 37. ed. Guanabara Koogan, Rio de Janeiro, 1995.

Dirección para correspondencia:

Prof. Dr. Mariano del Sol

Facultad de Medicina

Universidad de La Frontera

Casilla $54-\mathcal{D}$

Temuco - CHILE

Email:mdelsol@ufro.cl

Recibido : 24-04-2005

Aceptado: 27-07-2005 